\title{
Existence, uniqueness and monotonic behavior of the solution of classical flow distribution problem for hydraulic networks with pressure- dependent closure relations
}

\author{
Leonid Korelstein ${ }^{1, *}$ \\ ${ }^{1}$ PSRE Co, Moscow, Russia
}

\begin{abstract}
Existence, uniqueness and monotonic behavior of the solution of classical flow distribution problem for hydraulic networks with pressuredependent closure relations was proved. The closure relation can have very general form, restricted only by continuity and monotonicity conditions necessary for providing existence, uniqueness and continuity of flow distribution problem for each branch. It is shown that network as a whole "inherits" monotonicity and continuity of its branches behavior, and this provides existence and uniqueness of solution.
\end{abstract}

The question of existence and uniqueness of flow distribution always was in focus of interest of piping networks researchers ([1], [2], [3]). This problem was studied by Merenkov and Hasilev [4], and Suharev [5, 6]. The latest advanced research on the matter were done by Epifanov and Zorkaltsev, who prove existence and uniquesness of solution for the most general condition on edge closure relations for both classical flow distribution problem [7], and some non-classical variants $[8,9,10,11]$. In all these works the problem was reduced to optimization of strictly convex function.

Nevertheless all mentioned results were obtained for pressure-independent edge closure relations. Such assumption is quite reasonable for isothermal incompressible liquid flow, but not valid in many cases of real gas flow and especially for gas-liquid flow, fluid properties highly depend on pressure. In this case there are no evident way to effectively reduce the problem to optimization task [6]. But (as a rule) it is possible to use modified variants of classical algorithms for finding solutions for such networks можно эффективно (such modified algorithms proposed by Mikhailovsky and Novitsky [12, 13, 14]). But lack of mathematically accurate proof of uniqueness and existence of solution remained some filling of dissatisfaction - especially because it is known for long time, that in similar problems of non-isothermal flow, especially gas-liquid flow, solution can be not unique!

It found out that for this kind of networks great role is played by properties of monotonicity (they were studies in latest researches of Chertkov - see for example [15]). Using them, the author managed to prove existence and uniqueness of solution of classical

\footnotetext{
*Corresponding author: korelstein@ truboprovod.ru
} 
flow distribution problem for very general conditions on pressure-dependent edge closure relations, which are direct generalization of Epifanov and Zorkaltsev results [7-11].

Already after writing the first variant of the article the author found out that relation between monotonicity of network flow distribution solution, and existence and uniqueness of the solution was discovered almost 60 years ago first by famous hydraulic network researcher Birkghoff and his colleague Kellogg [16], and then in more general form by famous mathematician Rheinboldt [17]. The later established it for the most general case of «abstract» network in frame of his study of multi-dimensional mappings, correspondent to different matrix types. It is very strange, but these results found out to be not noticed by hydraulic network specialists (except articles $[18,19]$ of that time) and unjust forgotten. The author hopes that this article will help to restore historical justice and these ideas will became natural part of hydraulic network theory.

\section{Problem definition and closure relations}

Let $G$ - directed graph with $N_{V}$ nodes (node set $V$ ) and $N_{E}$ edges (edge set $E$ ). Flow rate $X_{i}$ on edge $\mathrm{i}$ is related with start and end pressures $P_{F i}$ and $P_{L i}$ by closure relation

$$
X_{i}=\varphi_{i}\left(P_{F i}, P_{L i}\right)
$$

Let $A$ - incidence matrix of graph $G ; Q$ - node inflow vector. Balance equations are

$$
\mathrm{AX}=\mathrm{Q}
$$

Using matrixes $A_{F}$ and $A_{L}$, correspondent to starting and ending edges $\left(A=A_{F}+A_{L}\right)$, vector $P$ and vector $\Phi$ of functions $\varphi_{i}$, (1) can be written as

$$
\mathrm{X}=\Phi\left(\mathrm{P}_{\mathrm{F}}, \mathrm{P}_{\mathrm{L}}\right), \mathrm{P}_{\mathrm{F}}=\mathrm{A}_{\mathrm{F}}^{\mathrm{T}} \mathrm{P}, \mathrm{P}_{\mathrm{L}}=-\mathrm{A}_{\mathrm{L}}^{\mathrm{T}} \mathrm{P}
$$

So we have $N_{V}+N_{E}$ equations for $2 N_{V}+N_{E}$ unknowns ( $P, Q$ and $X$ ). But equations (2) are not independent - for connected graph $G$ matrix $A$ has rank $N_{V}-1$, with additional equation:

$$
\sum_{\mathrm{i}=1}^{\mathrm{N}_{\mathrm{V}}} \mathrm{Q}_{\mathrm{i}}=0
$$

So for connected graph $G$ number of unknown is exceed by $N_{V}$ number of independent equations, so values of $N_{V}$ unknowns have to be set.

In classical flow distribution problem (CFDP) node pressure $P_{f i x}$ is set in $N_{P}>0$ nodes (set $V_{P}$ ) and inflow $Q_{f i x}$ is set in remaining $N_{Q}=N_{V}-N_{P}$ nodes (set $V_{Q}$ ); pressures $P_{v a r}$ are needed to be find in remaining $N_{Q}$ узлах, plus flow rates $X$ and inflows $Q_{v a r}$ in $N_{P}$ nodes with set pressure. The latest can be defined using equations (1) and (2), so it is enough to find $P_{\text {var. }}$.

For «traditional» hydraulic networks functions $\varphi_{i}$ depend only on pressure difference: $X_{i}=\varphi_{i}\left(P_{F i}-P_{L i}\right)$. Conditions were defined in [7] for functions $\varphi_{i}$ (or invert functions $f_{i}$ ), which provide existence and uniqueness of CFDP solution for «traditional» hydraulic networks (Conditions A): 1) Continuity; 2) Strict monotonic increase; 3 ) Definition on all $\mathbb{R}$; 4) $\varphi_{i}(y) \rightarrow+\infty$ when $y \rightarrow+\infty$ and $\varphi_{i}(y) \rightarrow-\infty$ when $y \rightarrow-\infty$.

Condition 2 is necessary for uniqueness of solution; condition 1 and 2 are very natural and often fits in engineering practice. While conditions 3 ) and 4) are convenient mathematical extrapolation, which provides to guarantee existence of solution for all set values of pressures and inflows - in practice region of possible pressure is restricted - almost always from below 
(as a minimum, pressures should be positive), but also often by upper limits (by technological conditions, strength demands etc), so possible flow rates and inflows values are also restricted. So (as noted in [10]) in practice it would be good to have estimations of limit on set pressures and inflows, for which CFDP has solution in frame of value restriction on arguments of functions $\varphi_{i}$ and $f_{i}$. But this is a separate task.

Set of functions, which fit conditions 1)-4), is noted as $\tilde{Z}_{a}$. It differs from introduced in [7-10] set $\tilde{Z}$ only by lack of conditions of zero pressure losses on zero flow rate. Set $\tilde{Z}_{a}$ can be created from $\tilde{Z}$ by addition of arbitrary constant. Functions from $\tilde{Z}$ correspond to passive edges, while functions from $\tilde{Z}_{a}$ also can represent active edges (with level difference, pumps, compressors etc).

Now let define set $\tilde{Z}_{a}^{2}$ of allowable pressure-dependent closure relations. It is natural generalization of $\tilde{Z}_{a}$. Function $\varphi \in \tilde{Z}_{a}^{2}$, if (Conditions A2): 1) $\varphi\left(P_{F}, P_{L}\right)$ is a continuous function of both variables $\left.P_{F}, P_{L} ; 2\right) \varphi\left(P_{F}, P_{L}\right)$ strictly decreases on $P_{L}$ for any $P_{F} ; 3$ ) $\varphi\left(P_{F}, P_{L}\right)$ strictly increases on $P_{F}$ for any $\left.P_{L} ; 4\right) \varphi\left(P_{F}, P_{L}\right)$ is defined on all $\left.\mathbb{R}^{2} ; 5\right)$ For any $P_{F}$ $\varphi\left(P_{F}, P_{L}\right) \rightarrow-\infty$ when $P_{L} \rightarrow+\infty, \varphi\left(P_{F}, P_{L}\right) \rightarrow+\infty$ when $P_{L} \rightarrow-\infty$; 6) For any $P_{L} \varphi\left(P_{F}, P_{L}\right) \rightarrow+\infty$ when $P_{F} \rightarrow+\infty, \varphi\left(P_{F}, P_{L}\right) \rightarrow-\infty$ when $P_{F} \rightarrow-\infty$.

So set $\widetilde{Z}_{a}^{2}$ consists of continuous function, which for any $P_{L} \in \tilde{Z}_{a}$ as functions of $P_{F}$ and for any $P_{F} \in-\tilde{Z}_{a}$ as function of $P_{L}$. Examples of such functions are given in [12-14].

Like in case of $\tilde{Z}_{a}$, conditions 1)-3) are physically natural - while conditions 4)-6) are mathematical extrapolation.

Subset $\tilde{Z}^{2}$ of set $\tilde{Z}_{a}^{2}$ are functions, for which $\varphi(P, P)=0$ for any $P$. Functions from $\tilde{Z}^{2}$ represent passive edges, while $\tilde{Z}_{a}^{2}$ covers also active ones.

Note that any pressure-independent closure relation which fits some condition from list A, also fits correspondent condition(s) from list A2. So all further results for pressuredependent networks also are valid for "traditional" networks.

Note also, that introduction of function $\varphi\left(P_{F}, P_{L}\right)$, which gives unique value of flow rate on edge for pair of start and end pressure, is already some assumption. There are (rear) situations, when this is not right in practice - for example for centrifugal pumps with nonmonotonic H-Q curve. However flow in this case can be unstable (so called pumping pompage).

\section{Auxiliary definitions and properties}

We will need to establish so properties of functions from $\tilde{Z}_{a}^{2}$.

Lets $\varphi \in \tilde{Z}_{a}^{2}$. The equations $\varphi\left(P_{F}, P\right)-X=0$ and $\varphi\left(P, P_{L}\right)-X=0$ on $P$ define some implicit functions $f_{L}\left(P_{F}, \mathrm{X}\right)$ and $f_{F}\left(P_{L}, \mathrm{X}\right)$, which calculate end pressure by start pressure and flow rate, and vice versa. By definition of $\tilde{Z}_{a}^{2}$ these functions are defined on all $\mathbb{R} \times \mathbb{R}$, monotonic on both arguments, and go to infinity when any argument goes to infinity. These functions also are continuous on both arguments. This follows from strict monotonicity of functions $\varphi\left(P_{F}, P\right)-X$ and $\varphi\left(P, P_{L}\right)-X$ of $P$, and special «non-differential» variant of implicit function theorem ([20], [21]).

Note also, that direct of any edge is important only to correctly write balance equations (to assign correct sign to flow rate). The direction of the edge always can be inverted by replacing closure relation of inverted edge with $\varphi^{*}\left(P_{F}, P_{L}\right)=-\varphi\left(P_{L}, P_{F}\right)$. 
Let $G$ - connected graph, on which CFDP is defined. Let define the following operation (P-reducing, or Pcut) - consider all nodes with set pressure, and split all of them with degree

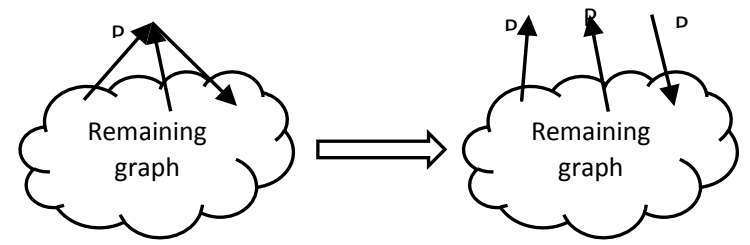

Fig. 1 $>1$ (Fig.1). As a result we will get graph $\operatorname{Pcut}(G)$, in which all nodes with set pressure have degree 1 (see example on fig.2). Graph Pcut $(G)$ cen be non-connected, let $G_{i P}$ - its connected subgraphs, and $G_{i}-$ sungraphs, from which they are produced (see example on Fig.2).

We will call $G_{i}-\mathrm{P}$ - components of graph $G$, and $G_{i P}$ - its P-reduced components. Pcomponents of graph intersect with each other only by nodes with set pressure, which connect them. We will call graph $G^{*}$ P-reduced, if it is connected and $\operatorname{Pcut}\left(G^{*}\right)=G^{*}$.

It is evident, that to solve CFDP on $G$ is the same as to solve it on each of $G_{i}$ or $G_{i P}$. Source data of CFDP influences on solution only locally - i.e. change of set inflow or pressure has influence only in P-components, containing specific node with set pressure or inflow. For node with set inflow this is the only P-component, node with set pressure can be part of several P-components. In other words, set pressure nodes split graph on independent parts.

All P-reduced graphs have special structure (simplifying analysis) - they are separate

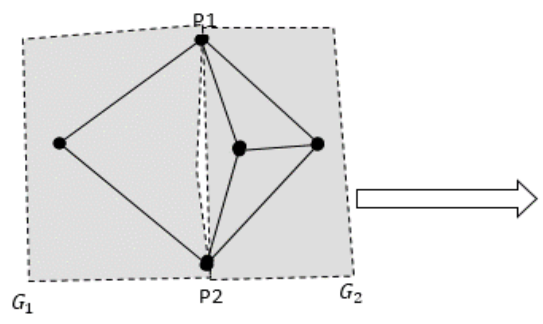

Fig. 2

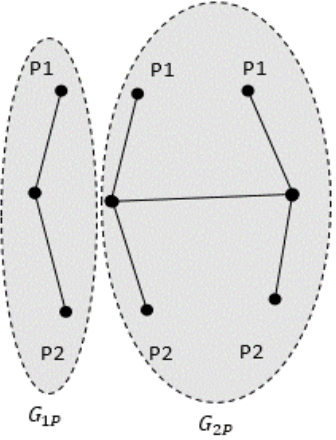

edges with set node pressures, or connected subgraph with nodes with set inflows and edges which connect them, plus connected degree 1 nodes with set pressure. P-components of graph have the same structure, the only difference is that nodes with set pressure can have degree $>1$.

Taking all said above into account, it is convenient to study properties of CFDP solution for P-reduced graphs - and then results usually can be easily generalized to any connected graph.

\section{Uniqueness and monotonicity of CFDP}

Let $\Omega \subseteq \mathbb{R}$ - some non-empty open connected set, and all functions $\varphi_{i}$ are defined on $\Omega \times \Omega$. $\Omega$ can be $\mathbb{R}$, or infinite open interval, or open interval (restricted by low and upper pressure boundaries). Let us first consider function $\varphi_{i}$, which are strictly monotonic, but not necessarily continuous. It found out that this is already enough to guarantee uniqueness and monotonicity of CFDP solution.

Let $P \in \Omega^{N_{V}}$ - node pressures vector. Then equations (1) and (2) completely define flow rates and nodal inflows. We will study how nodal pressures change influence on nodal inflows. 


\section{Lemma 1 (about nodal inflows).}

Let $G$ - connected graph with strictly monotonic edge closure relations, $P^{1}$ and $P^{2}-$ nodal pressure vectors, $X^{1}, X^{2}, Q^{1}, Q^{2}$ - flow rate and inflow vectors defined by (1) and (2). Let's consider the following sets of nodes: 1) $V^{+}$- set of nodes, for which $P_{i}^{2}>P_{i}^{1}$ (pressure increased); 2) $V^{-}$- set of nodes, for which $P_{i}^{2}<P_{i}^{1}$ (pressure decreased); 3) $V^{0}$ - set of nodes, for which $P_{i}^{2}=P_{i}^{1}$ (pressure didn't change); 4) $V^{0+}$ - subset of $V^{0}$ nodes, connected with any node from $V^{+}$, but not connected with any nodes from $\left.V^{-} ; 5\right) V^{0-}$ - subset of $V^{0}$ nodes, connected with any node from $V^{-}$, but not connected with any nodes from $V^{+}$;6) $V^{0 \pm}$ subset of $V^{0}$ nodes, connected with both nodes from $V^{+}$and $V^{-}$; 7) $V^{00}$ - subset of $V^{0}$ nodes, not connected with any node from $V^{+}$or $V^{-}$.

Then: 1) For any node $v \in V^{00} Q^{2}(v)=Q^{1}(v)$ (inflow doesn't change); 2) For any node $v \in V^{0+} Q^{2}(v)<Q^{1}(v)$ (inflow decreases); 3) For any node $v \in V^{0-} Q^{2}(v)>Q^{1}(v)$ (inflow increases); 4) If $V^{+} \neq \emptyset$ and $V^{+} \neq V$, then $\left.\sum_{v \in V^{+}} Q^{2}(v)>\sum_{v \in V^{+}} Q^{1}(v) ; 5\right)$ If $V^{-} \neq$ $\emptyset$ and $V^{-} \neq V$, then $\sum_{v \in V^{-}} Q^{2}(v)<\sum_{v \in V^{-}} Q^{1}(v)$.

Note, that it is impossible to say a priory anything about inflows in $V^{0 \pm}$ nodes.

Proof of Lemma 1.

Let $v$-graph node. For simplification we can change directions of connected edges so that they all start in $v$. According equation (1) inflow $Q(v)$ in $v$ is a sum of all flow rates in connected edges.

For $v \in V^{00}$, for all edges connected with $v$ end nodal pressures $P_{i}^{2}$ are equal $P_{i}^{1}$, so all flow rates also are equal, and $Q^{2}(v)=Q^{1}(v)$.

If $v \in V^{0+}$, for all edges started in $v$ start pressure doesn't change $\left(P^{2}(v)=P^{1}(v)\right)$, while end pressure in end node $v_{L}$ does not change $\left(P^{2}\left(v_{L}\right)=P^{1}\left(v_{L}\right)\right)$ or increases $\left(P^{2}\left(v_{L}\right)>P^{1}\left(v_{L}\right)\right)$. In first case flow rate doesn't change, in second case it decreases (as edge closure relation is monotonic). As $v \in V^{0+}$, there exist at least one edge with decreased flow rate. So $Q^{2}(v)<Q^{1}(v)$, which prove $2^{\text {nd }}$ item of lemma. $3^{\text {rd }}$ item proof is similar.

Let $V^{+} \neq \varnothing$ and $V^{+} \neq V$. Let us sum all equations (1) for nodes from $V^{+}$. In resulting sum flow rates on edges connected nodes from $V^{+}$, will nullify each other. As $V^{+} \neq V$ and graph is connected, set of edges, connected nodes from $V^{+}$with other nodes (from $V \backslash V^{+}$), is not empty. For simplicity let's change their direction if necessary, so all start nodes were from $V^{+}$, and end ones from $V \backslash V^{+}$. The sum of all inflows on all $V^{+}$nodes is equal to sum of flow rates on edges from $V^{+}$«outside»: $\sum_{v \in V^{+}} Q(v)=\sum_{v \in V^{+}, u \in V \backslash V^{+}} X\left(e_{v u}\right)$. On each of such edges transition from $P^{1}$ to $P^{2}$ increase start node pressure, while end node pressure decreases or does not change. As close relation are monotonic, this produces flow rate increase on each of the edges, and increase of flow rate sum - and this proves item 4 of the lemma. Item 5 can be proved in a similar way.

Now we will find out CFDP solution properties which follow from closure relations monotonicity.

Theorem 1 (Uniqueness of CFDP solution).

Let $G$ - connected graph with strictly monotonic edge closure relations. Then CFDP solution for $G$ is unique.

Proof of Theorem 1.

In special case, when $N_{P}=N_{V}$, theorem is obvious.

Let's consider case $0<N_{P}<N_{V}$, (i.e. $V_{P} \neq \emptyset_{\text {и }} V_{Q} \neq \varnothing$ ). Let's suppose that for the same sets $V_{P}$ and $V_{Q}$, and the same nodal pressures and inflows there exist 2 different solutions of CFDP (noted by indexes 1 and 2). Then this solution should have different vectors of nodal pressures. Let's apply lemma 1 . As in $V_{P}$ nodes pressures are set, $V^{+} \subseteq V_{Q}$, i.e. $V^{+} \neq V$. Similar $V^{-} \subseteq V_{Q}$, and $V^{-} \neq V$. If $V^{+} \neq \emptyset$, item 4 of lemma 1 gives $\sum_{v \in V^{+}} Q^{2}(v)>$ 
$\sum_{v \in V^{+}} Q^{1}(v)$. But this is impossible, as $V^{+} \subseteq V_{Q}$, and all inflows in $V_{Q}$ are set and don't change. So $V^{+}=\emptyset$. Similar way we can get $V^{-}=\emptyset$. But this means, that $V^{0}=V$, i.e. all nodal pressure of solutions 1 and 2 are equal, so all flow rates and inflows are also equal. This proves theorem 1 .

Now let's study how CFDP solution depends on change of set nodal pressures and inflows The following theorem generalizes properties established in [15] for "classical" network, to the networks with pressure-dependent closure relations.

Theorem 2 (Monotonicity of CFDP solution).

Let $G$ - connected graph with strictly monotonic edge closure relations, with 2 CFDP (problems 1 and 2) defined with the same non-empty sets $V_{P}$ and $V_{Q}$, and set nodal pressures $P_{f i x}^{(1)}$ and $P_{f i x}^{(2)}$, and set inflows $Q_{f i x}^{(1)}$ and $Q_{f i x}^{(2)}$, related as $P_{f i x}^{(1)} \leq P_{f i x}^{(2)}$ и $Q_{f i x}^{(1)} \leq Q_{f i x}^{(2)}$, and solutions of both problems exist . Let $V_{P}^{+}$and $V_{Q}^{+}$- node sets with set nodal pressures and inflows, for which inequalities are strict. Then: 1) For all P-components of $G$, where are nodes from $V_{P}^{+}$or $V_{Q}^{+}, \forall v \in V_{Q} P^{2}(v)>P^{1}(v)$. In other P-components $\forall v \in V_{Q} P^{2}(v)=P^{1}(v)$; 2) If $N_{P}=1$ and $V_{Q}^{+} \neq \varnothing$, then in the only node $v$ with set pressure $Q_{v a r}^{(1)}(v)>Q_{v a r}^{(2)}(v)$. Otherwise $Q_{v a r}^{(1)}(v)=Q_{v a r}^{(2)}(v)$; 3) If $N_{P}>1$, then $Q_{v a r}^{(1)}(v)>Q_{v a r}^{(2)}(v)$ for $\forall v \in V_{P} \backslash V_{P}^{+}$ from any P-component, containing nodes from $V_{Q}^{+} \cup V_{P}^{+}$. For other $\in V_{P} \backslash V_{P}^{+} Q_{v a r}^{(1)}(v)=$ $\left.Q_{v a r}^{(2)}(v) ; 4\right) \quad$ If $N_{P}>1, \quad V_{Q}^{+}=\emptyset, \quad V_{P}^{+} \neq \emptyset, \quad V_{P} \backslash V_{P}^{+} \neq \emptyset, \quad$ then $\quad \sum_{v \in V_{P}^{+}} Q_{v a r}^{(1)}(v)<$ $\sum_{v \in V_{P}^{+}} Q_{v a r}^{(2)}(v)$.

\section{Proof of Theorem 2.}

It is enough to prove the theorem for each P-component. In those of them, where there are no nodes from $V_{P}^{+}$or $V_{Q}^{+}$, CFDP data is the same, so according Theorem 1 the solution is the same - and this proves last parts of the theorem items 1 and 3.

Let $G^{*}$-P-component with nodes from $V_{P}^{+}$or $V_{Q}^{+}$. We will apply Lemma 1 for it.

For $G^{*} V_{P} \neq \varnothing$ and $V_{P} \subseteq V^{0} \cup V^{+}$, thus $V^{-} \neq V$. According item 5 of lemma 1 sum of inflows on all nodes from $V^{-}$must decrease. But this is impossible $-V^{-}$can include only nodes with set inflows, where inflows don't change! Thus $V^{-}=\emptyset$. So all nodes of $G^{*}$ are contained in $V^{0} \cup V^{+}$.

Suppose that $V_{Q} \cap V^{0} \neq \varnothing$. Consider subgraph $G^{* *}$ of nodes $V_{Q}$, nodes $V_{P}^{+}$and edges which connect them. As $G^{*}$ is P-component, subgraph $G^{* *}$ is connected. Then $V_{Q} \cap V^{0+} \neq$ $\emptyset$. But according lemma 1 in nodes $V_{Q} \cap V^{0+}$ inflows have to decrease - this contradicts the theorem conditions. Thus $V_{Q} \cap V^{0}=\varnothing$ и $V_{Q} \subset V^{+}$, which proves 1 st item of the theorem.

All above shows that $V^{0}=V_{P} \backslash V_{P}^{+}$. Further, $V^{00}=\varnothing$ - otherwise nodes $V^{00}$ would not be connected with other nodes of $G^{*}$. So $V_{P} \backslash V_{P}^{+}=V^{0+}$, and according Lemma 1 inflows in these nodes decrease, and this proves items 2 and 3 .

Item 4 evidently follows from item 3 and equation (4).

\section{Continuity of CFDP solution}

Now we will add continuity condition to strict monotonicity of functions $\varphi_{i}$ and will study what additional properties of CFDP solution it brings.

Note by $Y$ vector, contained with CFDP source data - first $N_{P}$ components - set nodal pressures $P_{f i x}$, other $N_{Q}$ - set inflows $Q_{f i x} ; Y \in \Omega^{N_{P}} \times \mathbb{R}^{N_{Q}}$. Note by $E$ set of $Y$, for which CFDP is solvable. What can we say about $E$ ?

Theorem 3 (Continuity and monotonicity of CFDP solution). 

Then:

Let $G$ - Connected graph with continuous and strictly monotonic edge closure relations.

1. $E$ is homeomorphic to $\Omega^{N_{V}}$ (or $\mathbb{R}^{N_{V}}$, what is the same) and thus non-empty, open and connected.

2. All solutions parameter (nodal pressures, flow rates, inflows) are continuous functions of source data.

3. Solution is monotonic on source data:

a. Pressures in nodes from $V_{Q}$ strictly increase when set inflows and nodal pressures increase in their P-component, and don't depend on source data in other Pcomponents.

b. Inflows in all nodes from $V_{P}$ strictly decrease when set inflows increase in their Pcomponents, and don't depend on set inflows in other P-components.

c. Inflow in node from $V_{P}$ :

i. Strictly increases with set pressure increases in the same node, if there are other nodes in $V_{P}$.

ii. Strictly decreases while set pressures in other nodes increase from the same Pcomponent.

iii. Does not change in all other cases.

Proof of theorem 3.

Consider mapping $\Psi: \Omega^{N_{V}} \rightarrow \Omega^{N_{P}} \times \mathbb{R}^{N_{Q}}$, which maps nodal pressure vector $P$ to vector $Y$, which consists from pressures $P_{i}$ in nodes from $V_{P}$ and inflows $Q_{i}$ in nodes from $V_{Q}$, calculated from $P$ using equations (1)-(2). In fact mapping $\Psi$ maps vector $P$ to source data of CFDP for which it is a solution. So $E=\Psi\left(\Omega^{N_{V}}\right)$.

As functions $\varphi_{i}$ are continuous, mapping $\Psi$ is also continuous. As functions $\varphi_{i}$ are strictly monotonic, according theorem 1 this mapping is also injective. So according Brouwer invariance of domain theorem, mapping $\Psi$ is homeomorphism, and its image $E=\Psi\left(\Omega^{N_{V}}\right)$ is open and homeomorphic to $\Omega^{N_{V}}$.

As $\Psi$ is homeomorphism, invert mapping $\Psi^{-1}$ is continuous on $E$. This means that nodal pressures continuously depend on source data of CFDP. As functions $\varphi_{i}$ are also continuous, flow rates and inflows (calculated by equations (1), (2)) also depends continuously on CFDP source data.

$3^{\text {rd }}$ item of the theorem directly follows from theorem 2 .

Theorem 4 (CFDP solution existence for intermediate source data).

Let $G$ - connected graph with strictly monotonic and continuous edge closure relations, and there is CFDP on $G$ with $N_{Q}>0$.

If $Y^{1}=\left(P_{f i x}^{1}, Q_{f i x}^{1}\right) \in E, Y^{2}=\left(P_{f i x}^{2}, Q_{f i x}^{2}\right) \in E, P_{f i x}^{1} \leq P_{f i x}^{2}, Q_{f i x}^{1} \leq Q_{f i x}^{2}$, and for $\left(P_{f i x}, Q_{f i x}\right) P_{f i x}^{1} \leq P_{f i x} \leq P_{f i x}^{2} Q_{f i x}^{1} \leq Q_{f i x} \leq Q_{f i x}^{2}$, then $\left(P_{f i x}, Q_{f i x}\right) \in E$ and for CFDP solution $P_{\text {var }}^{1} \leq P_{\text {var }} \leq P_{\text {var }}^{2}$.

In other words, if CFDP has is solvable for some "boundary" pressures and inflows, it is solvable for intermediate source data.

Proof of theorem 4.

Let $K$ - set $P_{\text {fix }}^{(1)} \leq P_{\text {fix }} \leq P_{\text {fix }}^{(2)}, Q_{\text {fix }}^{(1)} \leq Q_{f i x} \leq Q_{\text {fix }}^{(2)}$ in $\Omega^{N_{P}} \times \mathbb{R}^{N_{Q}}$. In fact we need to prove, that $K \subseteq E$, i.e. $K \backslash E=\emptyset$. Suppose that $K \backslash E \neq \emptyset$. As $K$ is compact, and $E$ is open, $K \backslash E$ is also compact. So $K \backslash E$ contains some point $Y^{*}$, nearest to $Y^{1}$. Consider interval $I^{*}=\left[Y^{1}, Y^{*}\right]$, connecting points $Y^{*}, Y^{1}$. As $Y^{*}$ is the nearest to $Y^{1}$ point in $K \backslash E$, all points of $I^{*}$, except $Y^{*}, \in E$. Consider mapping $\Psi^{-1}$ on interval $\left[Y^{1}, Y^{*}\right)$. According theorem 4 it is continuous, increases monotonically on all coordinates and upper limited by coordinates of $\Psi^{-1}\left(Y^{2}\right)$. So $\Psi^{-1}(Y)$ has some limit $P^{*}$, when $Y \rightarrow Y^{*}$ on $I^{*}$. $\Psi$ is continuous, so $\Psi\left(P^{*}\right)=$ 
$Y^{*}$, and $Y^{*} \in E-$ contradiction, which proves $K \backslash E=\emptyset$. Inequality $P_{v a r}^{1} \leq P_{v a r} \leq P_{v a r}^{2}$ follows from item $3 \mathrm{a}$ of theorem 4 .

\section{CFDP solution existence theorem}

\section{Theorem 5 (Existence of CFDP solution).}

Let $G$ - connected graph with all edges closure relation from $\tilde{Z}_{a}^{2}$, for which CFDP is defined. Then solution of such CFDP always exists.

Proof of theorem 5.

We will prove the theorem by induction on number of edges $N_{E}$ of graph $G$. In fact it will describe some recursive algorithm for finding the solution, which allows to establish its existence.

The base of induction $\left(N_{E}=1\right)$. For Graph with one edge (and two nodes), for the case, when pressure is set in all nodes, CFDP is solvable by definition. If pressure is set in one node and inflow in another one, CFDP solution exists, because finctions $f_{L}$ and $f_{F}$ for edges with closure relations from $\tilde{Z}_{a}^{2}$ are defined on all $\mathbb{R} \times \mathbb{R}$ (see section 2 ).

The step of induction. Let graph $G$ has $N_{E}>1$ edges and CSFP solution existence is proved for all graphs with edges number less than $N_{E}$. Consider graph $G^{\prime}=\operatorname{Pcut}(G)$. CSFP solution on graph $G$ is the same as on $G^{\prime}$. If the latest is non-connected and contains several connected subgraphs (P-reduced components), each of them contain $<N_{E}$ edges, so on each of them CSFP has solution, and union of these solutions gives solution on $G^{\prime}$.

The case remains when $G^{\prime}$ is connected and is P-reduced. Select some node $v^{\prime}$ with set pressure $P_{f i x}\left(v^{\prime}\right)$ in $G^{\prime}$. This node has degree 1 in the graph an is connected by edge $e^{\prime}$ with some node $v^{\prime \prime}$ with set inflows $Q_{f i x}\left(v^{\prime \prime}\right)$. For further simplification, we can change direction of $e^{\prime}$, if necessary, so it starts in $v^{\prime}$ and ends in $v^{\prime \prime}$. Let $G^{\prime \prime}$ - graph, produced from $G^{\prime}$ by deleting node $v^{\prime}$ and edge $e^{\prime}$ (Fig.3). It is connected and has $N_{E}-1$ edges, so on it any CFDP is solvable. There can be 2 cases - 1) $G^{\prime}$ has only one node with set pressure (node $v^{\prime}$ ); 2) $G^{\prime}$ has more than one node with set pressure - so $G^{\prime \prime}$ also contains nodes with set pressure.

1 st case. In this case we can do «direct calculation» of edge $e^{\prime}$.

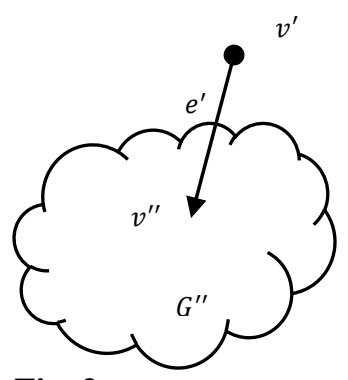

Fig. 3

From equation (4) we need to have $Q\left(v^{\prime}\right)=-\sum_{v \in G^{\prime}, v \neq v^{\prime}} Q_{f i x}(v)$. As $v^{\prime}$ has degree 1 , let take flow rate on $e^{\prime}$ equal to $X\left(e^{\prime}\right)=Q\left(v^{\prime}\right)$ and calculate pressure in $v^{\prime \prime}$ using $f_{L}$ for $e^{\prime}$ : $P\left(v^{\prime \prime}\right)=f_{e^{\prime} L}\left(P_{f i x}\left(v^{\prime}\right), X\left(e^{\prime}\right)\right)$. Set CFDP on $G^{\prime \prime}$ with the same source data, as on $G^{\prime}$, but instead of inflow in $v^{\prime \prime}$ let set pressure in it. Solution of such CFDP on $G^{\prime \prime}$ exists, and along with set pressure in $v^{\prime}$ will provide solution of original CFDP on $G^{\prime}$. This is evident for all nodes, except $v^{\prime \prime}$. In $v^{\prime \prime}$ for found solution $Q\left(v^{\prime \prime}\right)=-\sum_{v \in G^{\prime \prime}, v \neq v^{\prime \prime}} Q_{f i x}(v)-X\left(e^{\prime}\right)=$ $-\sum_{v \in G^{\prime \prime}, v \neq v^{\prime \prime}} Q_{f i x}(v)+\sum_{v \in G^{\prime}, v \neq v^{\prime}} Q_{f i x}(v)=Q_{f i x}\left(v^{\prime \prime}\right)$.

2nd case. In this case we will find such pressure $P^{\prime \prime}$ in $v^{\prime \prime}$, so CFDP on $G^{\prime \prime}$ with set pressure $P^{\prime \prime}$ in $v^{\prime \prime}$, and the same conditions in other nodes of $G^{\prime \prime}$, as on $G^{\prime}$, in combination with flow rate on $e^{\prime}$, correspondent to $P_{f i x}\left(v^{\prime}\right)$ and $P^{\prime \prime}$, would provide solution of original CFDP on $G^{\prime}$. To do this, we only need to have set inflow in $v^{\prime \prime}$, i.e. to have $Q_{G^{\prime \prime} \text { var }}\left(v^{\prime \prime}, P^{\prime \prime}\right)-\varphi_{e^{\prime}}\left(P_{f i x}\left(v^{\prime}\right), P^{\prime \prime}\right)=Q_{f i x}\left(v^{\prime \prime}\right)$, where $\varphi_{e^{\prime}}$ is function $\varphi$ for $e^{\prime}$, $Q_{G^{\prime \prime}}$ var $\left(v^{\prime \prime}, P^{\prime \prime}\right)$ - inflow in $v^{\prime \prime}$ for solution of CFDP on $G^{\prime \prime}$ with set pressure $P^{\prime \prime}$ in $v^{\prime \prime}$ and the same condition in other nodes. Consider function $q\left(P^{\prime \prime}\right)$ :

$$
q\left(P^{\prime \prime}\right)=Q_{G^{\prime \prime}} \operatorname{var}\left(v^{\prime \prime}, P^{\prime \prime}\right)-\varphi_{e^{\prime}}\left(P_{f i x}\left(v^{\prime}\right), P^{\prime \prime}\right)-Q_{f i x}\left(v^{\prime \prime}\right)
$$


Function $-\varphi_{e^{\prime}}\left(P_{f i x}\left(v^{\prime}\right), P^{\prime \prime}\right)$ of $P^{\prime \prime}$ is from set $\tilde{Z}_{a} \cdot Q_{G^{\prime \prime}}$ var $\left(v^{\prime \prime}, P^{\prime \prime}\right)$ as function of $P^{\prime \prime}$ is defined on all $\mathbb{R}$. According item 2 of theorem 3 it is continuous on $P^{\prime \prime}$, and according item 3 ci of theorem 3 increases monotonically. Thus, function $q\left(P^{\prime \prime}\right)$ also $\in \tilde{Z}_{a}$, so should equal 0 in some point. This point is the value of $P^{\prime \prime}$, which we are looking for.

\section{References}

1. B.N. Pshenichny, J. Calc. Mathemathics and Math. Physiscs, 5, 942 (1962) [in Russian]

2. M. Collins, L. Cooper, R. Helgarson, J. Kennington, L. Leblanc, Management Science, 24, 747 (1978)

3. A. Bermudes, J. Gonzalez-Diaz, F.J. Gonzalez-Dieguez, Non-linear analysis: Real World Applications, 37, 71(2017)

4. A.P. Merenkov, V.Y. Hasilev, Theory of Hydraulic Circuits (1985) [in Russian]

5. M.G. Suharev, Cybernetics, 6, 9 (1969) [in Russian]

6. M.G. Suharev, Piping Systems of Energetics: Control of development and operation, 15 (Novosibirsk, Nauka, 2004) [in Russian]

7. S.P. Epifanov, V.I. Zorkaltsev, Computational Technologies, 14 (1), 67 (2009) [in Russian]

8. S.P. Epifanov, V.I. Zorkaltsev, Sib. Zh. Ind. Math., 13 (4), 15 (2010) [in Russian]

9. S.P. Epifanov, V.I. Zorkaltsev, Izv. Vyssh. Uchebn. Zaved. Mat., 9, 76 (2010) [in Russian]

10. S.P. Epifanov, V.I. Zorkaltsev, Cybernetics and Systems Analysis, 47(1), 74 (2011) [in Russian]

11. S.P. Epifanov, V.I. Zorkaltsev, D.S. Medvezhonkov, Piping Systems of Energetics. Methodical and applied problems of mathematical simulation, 144 (Novosibirsk, Nauka, 2015) [in Russian]

12. E.A. Mikhailovsky, Piping Systems of Energetics. Mathematical and computer simulation, 34 (Novosibirsk, Nauka, 2014) [in Russian]

13. N.N. Novitsky, E.A. Mikhailovsky, Proceedings Mathematical models and methods of analysis and optimal synthesis of developing piping and hydraulic systems, 57 (Irkutsk, ESI SB RAS, 2014) [in Russian]

14. E.A. Mikhailovsky, N.N. Novitsky, St. Petersburg Polytechnical University J. Physics and Mathematics, 1 (2), 120 (2015) [in Russian]

15. Marc Vuffray, Sidhant Misra, Michael Chertkov, Monotonicity of Dissipative Flow Network Renders Robust Maximum Profit Problem Tractable: General Analysis and Application to Natural Gas Flow. https://arxiv.org/abs/1504.02370

16. Garrett Birkhoff, Bruce Kellogg, Proc. Of Symp. on Generalized Networks, 16, 443 (Published for Polyt. Inst. Brooklyn, N.Y.,1966)

17. Werner C. Rheinboldt, J. of Math. Analysis and applications, 32(2), 274 (1970)

18. T.A. Porsching, SIAM J. Numer. Anal, 6 (3), 437 (1969)

19. T.A. Porshing, Quarterly of Applied Mathematics, 34 (1), 47 (1976)

20. K. Jittorntrum, Journal of optimization theory and applications, 25 (4), 575 (1978)

21. S. Kumagai, Journal of optimization theory and applications, 31 (2), 285 (1980) 